\title{
Correction to: Telmisartan attenuates kidney apoptosis and autophagy-related protein expression levels in an intermittent hypoxia mouse model
}

\author{
Xiao-Bin Zhang ${ }^{1,2,3,4,5}$ (D) Jing-Huang Cai ${ }^{1,2} \cdot$ Yu-Yun Yang ${ }^{1,2} \cdot$ Yi-Ming Zeng $^{3,4,5} \cdot$ Hui-Qing Zeng ${ }^{1,2} \cdot$ Miao Wang $^{1,2} \cdot$ \\ Xiao Cheng ${ }^{1,2} \cdot$ Xiongbiao Luo $^{6} \cdot$ Henry Chidozie Ewurum ${ }^{6}$
}

Published online: 26 June 2020

(C) Springer Nature Switzerland AG 2020

\section{Correction to: Sleep and Breathing (2019) 23:341-348}

https://doi.org/10.1007/s11325-018-1720-9

In the article that appeared on Page: 341-348, Vol 23 (15 September 2018) of the Sleep and breathing [1], one error was discovered in Figure 3. The picture of Normoxia and $\mathrm{CIH}$ in $100 \mathrm{X}$ is the same one. The corrected version of Figure 3 is presented here.

\section{Reference}

1. Zhang XB, Cai JH, Yang YY et al (2019) Telmisartan attenuates kidney apoptosis and autophagy-related protein expression levels in an intermittent hypoxia mouse model. Sleep Breath 23:341-348

Publisher's note Springer Nature remains neutral with regard to jurisdictional claims in published maps and institutional affiliations.

The online version of the original article can be found at https://doi.org/ $10.1007 / \mathrm{s} 11325-018-1720-9$

Yi-Ming Zeng

ymzeng08@163.com

$\triangle$ Hui-Qing Zeng

zhq20071212@xmu.edu.cn

1 Department of Respiratory Medicine, Zhongshan Hospital, Xiamen University, No.201, Hubin Nan Road, Siming District,

Xiamen 361004, Fujian Province, People's Republic of China

2 Teaching Hospital of Fujian Medical University, Xiamen, China
3 Department of Pulmonary and Critical Care Medicine, The Second Affiliated Hospital of Fujian Medical University, No. 34, Zhongshanbei Road, Licheng District, Quanzhou 362000, Fujian Province, China

4 The Second Clinical Medical College of Fujian Medical University, Quanzhou, China

5 Center of Respiratory Medicine of Fujian Province, Quanzhou, China

6 Department of Computer Science, Xiamen University, Xiamen, Fujian, China 
Fig. 3 Kidney histopathological changes. The HE staining results illustrated that no abnormal architecture was found in all groups. CIH chronic intermittent hypoxia, HE hematoxylin and eosin staining
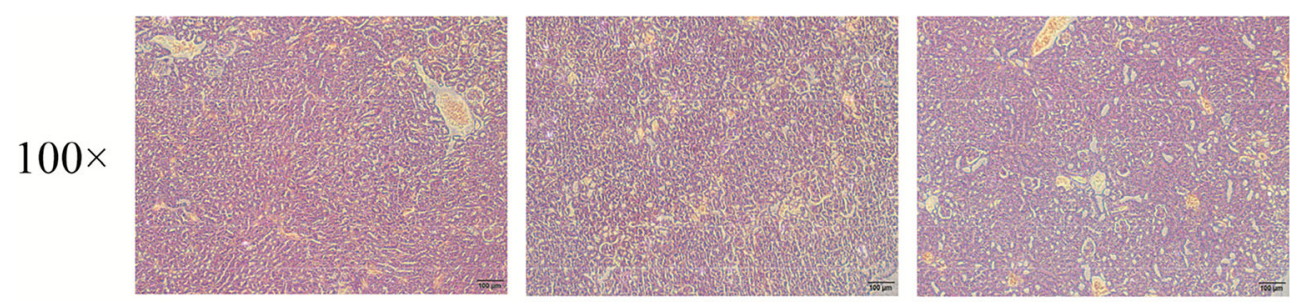

$400 \times$
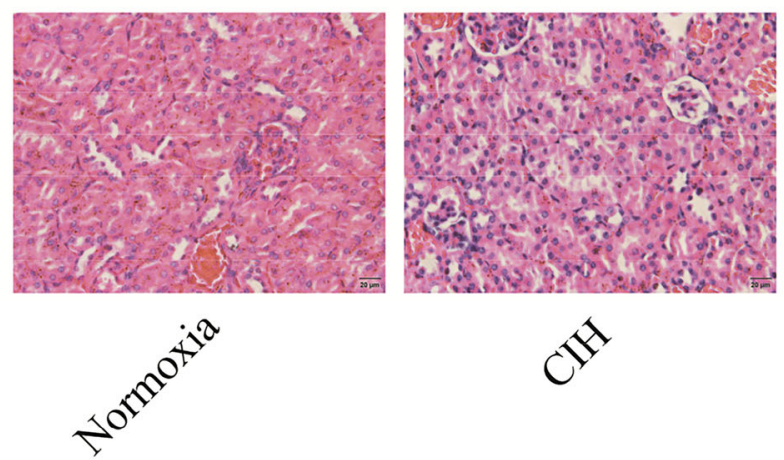
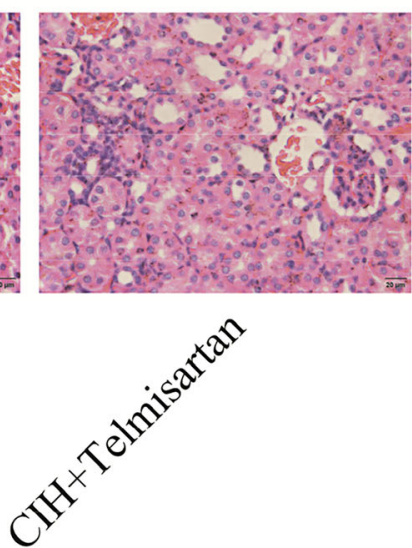